\title{
p-Adic String Compactified on a Torus
}

\author{
L. O. Chekhov and Yu. M. Zinoviev
}

Steklov Mathematical Institute, Vavilov st. 42, GSP-1, SU-117333 Moscow, USSR

\begin{abstract}
U(1)^{\times D}$ model with the Villain action on a $g$-loop generalization $F_{g}$ of the Bruhat-Tits tree for the $p$-adic linear group $G L\left(2, \mathbb{Q}_{p}\right)$ is considered. All correlation functions and the statistical sum are calculated. We compute also the averages of these correlation functions for $N$ vertices attached to the boundary of $F_{g}$. When the compactification radius tends to infinity the averages provide the $g$-loop $N$-point amplitudes of the uncompactified $p$-adic string theory, in particular for $g=0$ the Freund-Olson amplitudes.
\end{abstract}

\section{Introduction}

The idea of a non-archimedean string proposed in the papers $[1-4]$ has stimulated great activity in this field [5-9]. The authors of [3-4] have interpreted bosonic string amplitudes at the tree level of perturbation theory over the nonarchimedean number field $\mathbb{Q}_{p}$ as integrals of some combinations of multiplicative characters over $\mathbb{Q}_{p}$ (it is very close logically to the definition of the corresponding amplitudes for the usual open string over the real number field $\mathbb{R}$ ). In refs. [8] these $p$-adic amplitudes were produced from some non-local scalar field theory on $\mathbb{Q}_{p}$. Then the local formulation was given [9] which is actually more similar to the archimedean (Polyakov's) one. In the papers [9-10] the connection was established between $p$-adic string amplitudes and the Gaussian model on the Bruhat-Tits tree [11-14]. The Bruhat-Tits tree $T$ is manifestly determined to be the connected infinite graph with no loops, each vertex of $T$ being connected with exactly $p+1$ neighbour vertices by links. The branch $B_{z}$ is defined to be a connected subtree with the only boundary vertex $z$ of the graph $T \backslash B_{z}$ in the interior of $T$. By definition, the branch contains no cycles. A $g$-loop generalization of the $p$-adic string theory is given by the theory on the generalized tree $F_{g}$. It consists of a finite connected graph $F_{g}^{R}$ with $g$ independent loops, which is called a reduced graph, the branches $B_{x}, x \in F_{g}^{R}$, and each vertex is connected by links with exactly $p+1$ nearest neighbours (for every link two endpoints of which are identified with a vertex, we include the vertex itself twice into the number of its nearest neighbours). If the vertex $x \in F_{g}^{R}$ has only one nearest neighbour $y \in F_{g}^{R}, x \neq y$, then $p$ branches $B_{x}$ and 
the link $[x, y]$ form the branch $B_{y}$. Hence instead of the reduced graph $F_{g}^{R}$ we may consider the reduced graph $F_{g}^{\boldsymbol{R}} \backslash[x, y]$. From now on $\boldsymbol{F}_{0}^{\boldsymbol{R}}$ is merely a single vertex and $p+1$ branches should be added to $x$ in order to obtain the whole tree $T$. For $g>0$ every vertex $x \in F_{g}^{R}$ has $2 \leqq n(x) \leqq p+1$ nearest neighbours in $F_{g}^{R}$ and $b(x)$ $=p+1-n(x)$ branches should be added to $x$ in order to construct the generalized tree $F_{g}$. By Euler-Poincaré formula the reduced graph $F_{g}^{R}$ consists of $3 g-3$ or less segments (segment is the line containing only the vertices with exactly two nearest neighbours). The lengths of these segments are called the moduli of the corresponding $p$-adic surface, for examples see [10].

The open $p$-adic string world sheet $F_{0}=T$ may be interpreted as a coset space $P G L\left(2, \mathbb{Q}_{p}\right) / P G L\left(2, \mathbb{Z}_{p}\right)$, where $P G L(2, \mathbb{K})$ is the group of fractional linear transformations of the projective line $P^{1}(\mathbb{K})$ over a ring $\mathbb{K}$ (we deal with the field of $p$-adic numbers $\mathbb{Q}_{p}$ and with the ring of the $p$-adic integers $\left.\mathbb{Z}_{p}\right)[12,13]$. Usually the generalized tree $F_{g}$ may be interpreted as a coset space $F_{g}=T / \Gamma_{g}$, where $\Gamma_{g}$ is some Schottky group, i.e. a free subgroup of $P G L\left(2, \mathbb{Q}_{p}\right)$ with $g$ generators, all non-unit elements of which are hyperbolic [the element of $G L\left(2, \mathbb{Q}_{p}\right)$ is called hyperbolic if it has two eigenvalues which have different $p$-adic norms] [11-13]. The boundary of this world sheet corresponds to $p$-adic Mumford curve of genus $g$ [11]. The appropriate local string action on $F_{g}$ [for the scalar field $\phi(z) \in \mathbb{R}$ taking real values on the vertices $z \in F_{g}$ ] has the form:

$$
S(\phi)=-\beta / 2 \sum_{|x-y|=1}(\phi(x)-\phi(y))^{2} .
$$

All correlation functions for the action (1.1) were computed in [15]. In [10] the multiloop amplitudes with the action (1.1) were calculated for the scattering of $N$ identical tachyons attached to $\partial F_{g}$. In this paper we generalize the technique developed in $[9,10]$ to fields $\phi(z)$ taking values in a compact abelian group $U(1)^{\times D}$. The case $U(1)^{\times D}$ corresponds to the bosonic field compactified on the $D$-dimensional torus. Moreover it is of special interest since the compactified bosonic field appears in the archimedean string theory in the framework of the bosonization procedure [16]. In order to find the correlation functions, scattering amplitudes and statistical sum we shall use the results of the papers [17] where the general technique for the abelian gauge field theories on arbitrary lattices was presented. As the compactification radius tends to infinity we obtain the correlation functions and the amplitudes of the uncompactified $p$-adic string theory.

\section{Correlation Functions}

In order to find the correlation functions and the statistical sum for the infinite graph $F_{g}$ we calculate them first on a finite connected subgraph $K \subset F_{g}$ and take a limit $K \rightarrow F_{g}$. We suppose that a subgraph $K$ contains the reduced graph $F_{g}^{R}$. The subgraph $K$ may be considered as a finite cell complex. It consists of zero- and onedimensional cells: vertices and links. Every cell $\pm s_{i}^{q}$ is labelled by the integer $q=0,1$ (dimension) and by the number \pm 1 (orientation). The cells with the opposite orientation $s^{q}$ and $-s^{q}$ both belong to the lattice $K$. An integer-valued 
odd $\left(c^{q}\left(-s_{i}^{q}\right)=-c^{q}\left(s_{i}^{q}\right)\right)$ function $c^{q}$ on the $q$-dimensional cells is called a $q$-chain of the complex $K . c^{q}$ can be regarded as a formal sum $\sum m_{i} s_{i}^{q}$, where the integers $m_{i}=c^{q}\left(s_{i}^{q}\right)$. A set of $q$-chains is an Abelian group: $c^{q}+c^{\prime q}=\sum\left(m_{i}+m_{i}^{\prime}\right) s_{i}^{q}$. It is denoted by $C^{q}(K, \mathbb{Z})$. It is possible to introduce the inner product on $C^{q}(K, \mathbb{Z}):\left\langle c^{q}, c^{q}\right\rangle=\sum m_{i} m_{i}^{\prime}$. We define the boundary operator $\partial$ on $C^{q}(K, \mathbb{Z})$ by $\partial s^{0}=0$ and $\partial\left[x_{i}, x_{j}\right]=x_{j}-x_{i}$. By linearity it is easy to extend the boundary operator on $C^{q}(K, \mathbb{Z})$. We define the coboundary operator $\partial^{*}$ by the following relations $\partial^{*} c^{1}=0$ and $\left\langle\partial^{*} c^{0}, c^{1}\right\rangle=\left\langle c^{0}, \partial\left(c^{1}\right)\right\rangle$. A kernel $Z_{1}(K, \mathbb{Z})$ of a homomorphism $\partial: C^{1}(K, \mathbb{Z}) \rightarrow C^{0}(K, \mathbb{Z})$ is called a group of cycles of the complex $K$. The image $B_{0}(K, \mathbb{Z})$ of a homomorphism $\partial: C^{1}(K, \mathbb{Z}) \rightarrow C^{0}(K, \mathbb{Z})$ is called a group of boundaries of the complex $K$. The image $B^{1}(K, \mathbb{Z})$ of a homomorphism $\partial^{*}: C^{0}(K, \mathbb{Z}) \rightarrow C^{1}(K, \mathbb{Z})$ is called a group of coboundaries of the complex $K$.

A homomorphism of $C^{q}(K, \mathbb{Z})$ into an Abelian group $G$ is a $q$-chain of the complex $K$ with coefficients in $G$. A set of all these homomorphisms is an Abelian group which is denoted by $C^{q}(K, G)$. Each homomorphism $h^{q} \in C^{q}(K, G)$ is defined by its values on the $q$-chains $1 \cdot s_{i}^{q} \in C^{q}(K, \mathbb{Z})$ i.e. on the cells $s_{i}^{q}$. Thus $h^{q}$ is a $G$-valued function on the $q$-dimensional cells of $K$. On $C^{q}(K, G)$ we introduce the boundary and coboundary operators: $\partial h^{1}\left(c^{0}\right)=h^{1}\left(\partial^{*} c^{0}\right)$ and $\partial^{*} h^{0}\left(c^{1}\right)=h^{0}\left(\partial c^{1}\right)$. For example $\partial^{*} h^{0}([x, y])=h^{0}(x)^{-1} h^{0}(y)$. The group of cycles $Z_{1}(K, G)$, the groups of boundaries $B_{0}(K, G)$ and coboundaries $B^{1}(K, G)$ are defined in an obvious way. We consider two cases $G=U(1)^{\times D}, \mathbb{Z}^{D}$. The group $\mathbb{Z}^{D}$ is dual for the group $U(1)^{\times D}$ : if $\vartheta^{\mu} \in U(1)^{\times D}$ and $m^{\mu} \in \mathbb{Z}^{D}$, then

$$
\left\langle m^{\mu} \mid \vartheta^{\mu}\right\rangle=\prod_{\mu=1}^{D}\left(\vartheta^{\mu}\right)^{m^{\mu}}
$$

Analogously, the group $C^{q}\left(K, \mathbb{Z}^{D}\right)$ is dual for the group $C^{q}\left(K, U(1)^{\times D}\right)$ : if $\chi^{q} \in C^{q}\left(K, \mathbb{Z}^{D}\right)$ and $\varphi^{q} \in C^{q}\left(K, U(1)^{\times D}\right)$, then

$$
\left\langle\chi^{q} \mid \varphi^{q}\right\rangle=\prod_{s_{i}^{q} \in K}\left\langle\chi^{q}\left(s_{i}^{q}\right) \mid \varphi^{q}\left(s_{i}^{q}\right)\right\rangle,
$$

where multiplication runs over all positively oriented links of the lattice $K$.

Let us consider the $U(1)^{\times D}$-model on the lattice $K$. A field is a chain $\varphi^{0} \in C^{0}\left(K, U(1)^{\times D}\right)$, i.e. a function on the vertices of $K$ taking the values in $D$-dimensional torus $U(1)^{\times D}$. The key question is the choice of the action. It is possible to choose a zero-dimensional version of the Wilson action [18]. The most natural one is, in our opinion, a Villain action [19]:

$$
S\left(\varphi^{0}\right)=\sum_{s i \in K} \sum_{\mu=1}^{D} h\left(\left(\partial^{*} \varphi^{0}\left(s_{i}^{1}\right)\right)^{\mu}\right),
$$

where summing runs over all positively oriented links of the lattice $K$ and the function

$$
h\left(e^{i \frac{\theta}{r}}\right)=\ln \left[\sum_{m \in \mathbb{Z}} \exp \left\{-\frac{1}{2 \alpha^{2}}(\theta-2 \pi m r)^{2}\right\}\right] .
$$

The element of $U(1)$ is parametrized by $e^{i \theta / r}, \theta \in[0,2 \pi r[, r$ being the compactification radius. The statistical sum is

$$
Z_{K}=\int_{C_{(K, U(1) D)}^{0}} d \varphi^{0} e^{S\left(\varphi^{0}\right)},
$$


where $d \varphi^{0}$ is a normalized Haar measure on the compact group $C^{0}\left(K, U(1)^{D}\right)$. The correlation function has the form

$$
W_{K}\left(\chi^{0}\right)=\frac{1}{Z_{K}} \int_{C^{0}(K, U(1) \times D)}\left\langle\chi^{0} \mid \varphi^{0}\right\rangle e^{S\left(\varphi^{0}\right)} d \varphi^{0},
$$

where a chain $\chi^{0} \in C^{0}\left(K, \mathbb{Z}^{D}\right)$ is a character of the group $C^{0}\left(K, U(1)^{\times D}\right)$.

By Lemma 1 of [17] the correlation function $W_{K}\left(\chi^{0}\right)$ isn't zero only for boundaries $\chi^{0}=\partial \chi^{1}$ and

$$
W_{K}\left(\partial \chi^{1}\right)=\frac{1}{Z_{K}} \int_{B^{1}\left(K, U(1)^{\times D}\right)} d \varphi^{1}\left\langle\chi^{1} \mid \varphi^{1}\right\rangle \exp \left\{\sum_{s_{i}^{1} \in K} \sum_{\mu=1}^{D} h\left(\left(\varphi^{1}\left(s_{i}^{1}\right)\right)^{\mu}\right)\right\},
$$

where $d \varphi^{1}$ is a normalized Haar measure on the compact group $B^{1}\left(K, U(1)^{\times D}\right)$.

It is easy to compute the Fourier transform of the function $h\left(e^{i \vartheta}\right)$,

$$
1 /(2 \pi) \int_{0}^{2 \pi} e^{i n \vartheta} h\left(e^{i \vartheta}\right) d \vartheta=\frac{\alpha / r}{\sqrt{2 \pi}} \exp \left\{-\frac{\alpha^{2} n^{2}}{2 r^{2}}\right\} .
$$

By using the Fourier transformation on the group $B^{1}\left(K, U(1)^{\times D}\right)$, due to the formula (2.8) and Proposition 1 of [17] we obtain

$$
\begin{aligned}
& W_{K}\left(\partial \chi^{1}\right)=\frac{1}{Z_{k}}\left(\frac{\alpha^{2}}{2 \pi r^{2}}\right)^{g D / 2} \\
& \times \sum_{\left\{n_{i}^{\mu}\right\} \in \mathbb{Z}^{g \times D}} \exp \left\{-\frac{\alpha^{2}}{2 r^{2}} \sum_{\mu=1}^{D}\left\langle\left(\chi^{1}\right)^{\mu}+\sum_{i=1}^{g} n_{i}^{\mu} z_{i},\left(\chi^{1}\right)^{\mu}+\sum_{i=1}^{g} n_{i}^{\mu} z_{i}\right\rangle\right\},
\end{aligned}
$$

where $\chi^{1} \in C^{1}\left(K, \mathbb{Z}^{D}\right)$ and $\left(\chi^{1}\right)^{\mu}=\sum\left(\chi^{1}\left(s_{i}^{1}\right)\right)^{\mu} s_{i}^{1}$ is a chain from $C^{1}(K, \mathbb{Z}) . z_{1}, \ldots, z_{g}$ form a basis of the group of cycles $Z_{1}(K, \mathbb{Z})$. Since a subgraph $K$ contains the reduced graph $F_{g}^{R}$ and any branch has no cycles, the group $Z_{1}(K, \mathbb{Z})$ coincides with the group $Z_{1}\left(F_{g}^{R}, \mathbb{Z}\right)$. A subgraph $K$ is connected, hence any chain $\chi^{1} \in C^{1}\left(K, \mathbb{Z}^{D}\right)$ may be presented up to the shift $\left(\chi^{1}\right)^{\mu} \rightarrow\left(\chi^{1}\right)^{\mu}+\sum k_{i}^{\mu} z_{i}$ as $\left(\chi^{1}\right)^{\mu}=\sum_{j=1}^{N} m_{i}^{\mu} \mathscr{X}_{c, x_{i}}$, where $\mathscr{X}_{c, x_{i}}$ is some path without returns from the arbitrary fixed vertex $c \in F_{g}^{R}$ to the vertex $x_{i}$ and the integers $m_{j}^{\mu}$ satisfy the conditions for $\mu=1, \ldots, D$ :

$$
\sum_{j=1}^{N} m^{\mu}=0 \text {. }
$$

Taking the trivial chain $\chi^{1}=0$, i.e. $m_{j}^{\mu}=0$, we obtain the statistical sum

$$
Z_{K}=\left(\frac{\alpha^{2}}{2 \pi r^{2}}\right)^{g D / 2} \sum_{\left\{n_{i}^{\mu}\right\} \in \mathbb{Z}^{g \times D}} \exp \left\{-\frac{\alpha^{2}}{2 r^{2}} \sum_{i, j=1}^{g}\left(n_{i}, n_{j}\right)\left\langle z_{i}, z_{j}\right\rangle\right\},
$$

where the scalar product $\left(n_{i}, n_{j}\right)=\sum_{\mu=1}^{D} n_{i}^{\mu} n_{j}^{\mu}$. The symmetric $g \times g$ matrix of cycles of the graph $F_{g}^{R} \subset K$ :

$$
\Lambda_{i j}=\left\langle z_{i}, z_{j}\right\rangle
$$

is a natural non-archimedean analog of imaginary part of period matrix $2 \pi \operatorname{Im} \tau_{i j}$ in the archimedean case (note that in the open string case $\tau_{i j}$ is pure imaginary). 
Applying the Poisson resummation formula:

$$
\sum_{n} f(n)=\sum_{n} \int d x f(x) e^{2 \pi i n x}
$$

we can rewrite the relation (2.9) as

$$
\begin{aligned}
& W_{K}\left(\sum_{j=1}^{N} m_{j}^{\mu} x_{j}\right)=[\operatorname{det} \Lambda]^{-D / 2} W_{R}\left(\sum_{j=1}^{N} m_{j}^{\mu} x_{j}\right) \\
& \quad \times \theta\left(\sum_{k=1}^{g} \sum_{j=1}^{N} m_{j}^{\mu} \Lambda_{i k}^{-1}\left\langle\mathscr{X}_{c, x_{j}}, z_{k}\right\rangle \mid 2 \pi i \frac{r^{2}}{\alpha^{2}} \Lambda^{-1} \otimes I\right),
\end{aligned}
$$

where

$$
\begin{gathered}
W_{R}\left(\sum_{j=1}^{N} m_{j}^{\mu} x_{j}\right)=\exp \left\{-\frac{\alpha^{2}}{2 r^{2}}\left[\sum_{i, j=1}^{N}\left(m_{i}, m_{j}\right)\left\langle\mathscr{X}_{c, x_{i}}, \mathscr{X}_{c, x_{j}}\right\rangle\right.\right. \\
\left.\left.-\sum_{i, j=1}^{N} \sum_{k, l=1}^{g}\left(m_{i}, m_{j}\right)\left\langle\mathscr{X}_{c, x_{i}}, z_{k}\right\rangle \Lambda_{k l}^{-1}\left\langle\mathscr{X}_{c, x_{j}}, z_{l}\right\rangle\right]\right\}
\end{gathered}
$$

is the correlation function which is calculated for the theory with the action (1.1) on the lattice $K$ in the paper [15] and $\theta$-function [20],

$$
\theta\left(y_{i}^{\mu} \mid \Omega\right)=\sum_{\left\{n_{i}^{\mu}\right\} \in \mathbb{Z}^{g \times D}} \exp \left\{i \pi \sum_{\mu, v=1}^{D} \sum_{i, j=1}^{g} n_{i}^{\mu} \Omega_{i j}^{\mu v} n_{j}^{v}+2 \pi i \sum_{\mu=1}^{D} \sum_{i=1}^{g} n_{i}^{\mu} y_{i}^{\mu}\right\}
$$

depends on the vector $\left\{y_{i}^{\mu}\right\} \in \mathbb{C}^{g \times D}$ and on the symmetric complex $(g D) \times(g D)$ matrix $\Omega$ with the positively definite imaginary part. In our case $\Omega=2 \pi i \frac{r^{2}}{\alpha^{2}} \Lambda^{-1} \otimes I$, where $I$ is the identity $D \times D$ matrix.

Taking the trivial chain $m_{i}^{\mu}=0$ we obtain the statistical sum:

$$
Z_{K}=[\operatorname{det} \Lambda]^{-D / 2} \theta\left(0 \mid 2 \pi i \frac{r^{2}}{\alpha^{2}} \Lambda^{-1} \otimes I\right),
$$

$\theta(0 \mid \cdot)$ is the modular form of the weight $1 / 2$ with the transformation property:

$$
\theta\left(0 \mid i \tau^{-1}\right)=(\operatorname{det} \tau)^{1 / 2} \theta(0 \mid i \tau),
$$

and the modular parameter $\tau_{i j}^{\mu \nu}=2 \pi \frac{r^{2}}{\alpha^{2}} \Lambda_{i j}^{-1} \otimes I^{\mu \nu}$.

The result (2.13)-(2.16) was obtained in [15] for the zero-dimensional version of the Wilson action [19] but only in the weak-coupling region. If the vertices $x_{1}, \ldots, x_{N}$ are fixed, then the limit $K \rightarrow F_{g}$ for the correlation function and the statistical sum is given by the formulas (2.13)-(2.16). We denote these limits $W\left(\sum_{j=1}^{N} m_{j}^{\mu} x_{j}\right)$ and $Z$.

\section{Amplitudes}

Now we shall present the amplitude for the emission of $N$ particles from the boundary of $F_{g}$. 
Let us define the boundary of a generalized Bruhat-Tits tree $F_{g:}$ If a vertex $x \notin F_{g}^{R}$, then by the definition of the graph $F_{g}$ there exists the unique vertex $x^{R} \in F_{g}^{R}$ such that the path

$$
\mathscr{X}_{c, x}=\mathscr{X}_{c, x^{R}}+\mathscr{X}_{x^{R}, x},
$$

where the path $\mathscr{X}_{c, x_{i}^{R}}$ belongs to the reduced graph $F_{g}^{R}$ and the unique path $\mathscr{X}_{x^{R}, x}$ lies in the branch $B_{x^{R}}$. Any half-infinite path without returns in $B_{x^{R}}$ starting at the vertex $x^{R}$ we call a ray $x^{R} \rightarrow x$. The set of all rays will be called the boundary $\partial F_{g}$ of the generalized Bruhat-Tits tree $F_{g}$. On $\partial F_{g}$ we introduce the basis of open sets $\partial B_{x}$, where $x \in F_{g} \backslash F_{g}^{R}$, and $\partial B_{x}$ consists of all rays having infinite intersections with the branch $B_{x}$. The measure $\mu_{0}$ on $\partial F_{g}$ is defined by the following relation:

$$
\mu_{0}\left(\partial B_{x}\right)=p^{-\left\langle\mathscr{X}_{x}, x, \mathscr{X}_{\left.x^{R}, x\right\rangle}\right.} .
$$

The relation

$$
\left|x^{R} \rightarrow x, y^{R} \rightarrow y\right|_{p}=p^{-\left\langle x^{R} \rightarrow x, y^{R} \rightarrow y\right\rangle}
$$

defines the distance on $\partial F_{g}$.

The reduced graph $F_{0}^{R}$ is merely a single vertex $c$. The boundary $\partial F_{0}$ can be naturally identified with the $p$-adic projective line $P^{1}\left(\mathbb{Q}_{p}\right)[12,14]$ with the measure $d \mu_{0}$ related to the Haar measure $d x$ on $\mathbb{Q}_{p}$ by:

$$
\begin{aligned}
& d \mu_{0}(c \rightarrow x)=d x, \quad|x|_{p} \leqq 1, \\
& d \mu_{0}(c \rightarrow x)=d x /|x|_{p}^{2}, \quad|x|_{p}>1,
\end{aligned}
$$

where $|\cdot|_{p}$ is the standard $p$-adic norm on $\mathbb{Q}_{p}$, and the distance $|x, y|_{p}$ on $P^{1}\left(\mathbb{Q}_{p}\right)$ is defined by its restriction on $\mathbb{Q}_{p}$ :

$$
\begin{gathered}
|c \rightarrow x, c \rightarrow y|_{p}=|x-y|_{p},|x|_{p} \leqq 1,|y|_{p} \leqq 1 ; \\
|c \rightarrow x, c \rightarrow y|_{p}=\left|x^{-1}-y^{-1}\right|_{p},|x|_{p}>1,|y|_{p}>1 ; \\
|c \rightarrow x, c \rightarrow y|_{p}=1, \quad \text { otherwise. }
\end{gathered}
$$

We call the vertex $x^{R} \in F_{g}^{R}$ external if $x^{R}$ is the end of $b\left(x^{R}\right)>0$ branches in $F_{g}$. For $g>0$ by definition of the reduced graph $F_{g}^{R} 0<b\left(x^{R}\right) \leqq p-1$. The ray $x^{R} \rightarrow x$ starting at the external vertex $x^{R} \in F_{g}^{R}$ may be identified with the $p$-adic integer $x \in \mathbb{Z}_{p}$ of the form

$$
x=a_{0}+a_{1} p+a_{2} p^{2}+\ldots,
$$

where $0 \leqq a_{0} \leqq b\left(x^{R}\right)-1$ and $0 \leqq a_{i} \leqq p-1$ for $i>0$. We denote the set of these numbers as $\mathbb{Z}_{p}\left[a_{0}<b\left(x^{R}\right)\right]$. Thus for $g>0$,

$$
\partial F_{g} \cong \bigcup_{\substack{x^{R} \in F^{R} \\ b\left(x^{R}\right)>0}} \mathbb{Z}_{p}\left[a_{0}<b\left(x^{R}\right)\right] .
$$

It is easy to verify that under this correspondence

$$
\begin{gathered}
\left|x^{R} \rightarrow x, y^{R} \rightarrow y\right|_{p}=\left(|x-y|_{p}\right)^{\delta_{x^{R}, y^{R}}}, \\
d \mu_{0}\left(x^{R} \rightarrow x\right)=d x .
\end{gathered}
$$

We call the boundary $\partial K$ of the graph $K \subset F_{g}$ the set of all vertices from $K$ which have among the nearest neighbours the vertices from $F_{g} \backslash K$. Let 
$f_{1}\left(x^{R} \rightarrow x\right), \ldots, f_{N}\left(x^{R} \rightarrow x\right)$ be the positive continuous functions summable with the measure $d \mu_{0}\left(x^{R} \rightarrow x\right)$ on $\partial F_{g}$ defined by the relation (3.2). We define the amplitude

$$
A_{N}\left(m_{1}^{\mu}, \ldots, m_{N}^{\mu} \mid f_{1}, \ldots, f_{N}\right)=\lim _{\substack{K_{j} \rightarrow F_{g} \\ j=1, \ldots, N}} \sum_{\left\{x_{j} \in \partial K_{j}\right\}} W\left(\sum_{j=1}^{N} m_{j}^{\mu} x_{j}\right) \prod_{j=1}^{N} \bar{f}_{j}\left(x_{j}\right),
$$

where

$$
\bar{f}_{j}\left(x_{j}\right)=\left(\mu_{0}\left(\partial B_{x_{j}}\right)\right)^{-1} \int_{\partial B_{x_{j}}} d \mu_{0}\left(x^{R} \rightarrow x\right) f_{j}\left(x^{R} \rightarrow x\right) .
$$

If all functions $f_{j}\left(x^{R} \rightarrow x\right)=1$ then the definition of the amplitudes (3.10), (3.11) coincides with the definition of the $p$-adic string amplitudes of the papers $[8,9]$.

We find the limit (3.10) for the special sequence of graphs $\left\{K_{l}\right\}$ such that $\partial K_{l}$ $=\left\{x \in F_{g} \mid\left\langle\mathscr{X}_{x^{R}, x}, \mathscr{X}_{x^{R}, x}\right\rangle=l, x \in B_{x^{R}}, x^{R} \in F_{g}^{R}, b\left(x^{R}\right)>0\right\}$. We suppose also that the supports of the functions $f_{i}\left(x^{R} \rightarrow x\right)$ and $f_{j}\left(x^{R} \rightarrow x\right)$ do not intersect for $i \neq j$.

For $g=0$ the relations (2.13)-(2.16), (3.2), and (3.10), (3.11) imply

$$
\begin{aligned}
& A_{N}\left(m_{1}^{\mu}, \ldots, m_{N}^{\mu} \mid f_{1}, \ldots, f_{N}\right)=\lim _{\substack{l_{j} \rightarrow \infty \\
j=1, \ldots, N}} \exp \left\{\sum_{i=1}^{N}\left(\ln p-\frac{\alpha^{2}}{2 r^{2}}\left(m_{i}, m_{i}\right)\right) l_{i}\right\} \\
& \times \sum_{\left\{x_{j} \in \partial K_{K_{j}}\right\}}\left(\prod_{j=1}^{N} \int_{\partial B_{x_{j}}} d \mu_{0}(c \rightarrow x) f_{j}(c \rightarrow x)\right) \\
& \quad \times \exp \left\{-\frac{\alpha^{2}}{r^{2}} \sum_{i<j}^{N}\left(m_{i}, m_{j}\right)\left\langle\mathscr{X}_{c, x_{i}}, \mathscr{X}_{c, x_{j}}\right\rangle\right\} .
\end{aligned}
$$

Since the supports of the functions $f_{i}\left(x^{R} \rightarrow x\right)$ and $f_{j}\left(x^{R} \rightarrow x\right)$ do not intersect for $i \neq j$ the last sum in (3.12) absolutely converges as $l_{j} \rightarrow \infty$ to

$$
\int_{P^{1}\left(\mathbb{Q}_{p}\right)^{\times N}} f_{1}\left(x_{1}\right) d \mu_{0}\left(x_{1}\right) \ldots f_{N}\left(x_{N}\right) d \mu_{0}\left(x_{N}\right) \prod_{i<j}^{N} \mid x_{i}, x_{j} \frac{\alpha^{2}}{r_{p}^{2} \ln p}\left(m_{i}, m_{j}\right) .
$$

Here we use the relation (3.3) and we denote by $d \mu_{0}(x)$ the measure given by the right-hand sides of the relations (3.4). The distance $|x, y|_{p}$ is given by the right-hand sides of the relations (3.5). We also use the correspondence $\partial F_{0} \cong P^{1}\left(\mathbb{Q}_{p}\right)[12,14]$ and replace the functions $f_{j}(c \rightarrow x)$ simply by $f_{j}(x)$.

It follows now from (3.12) that

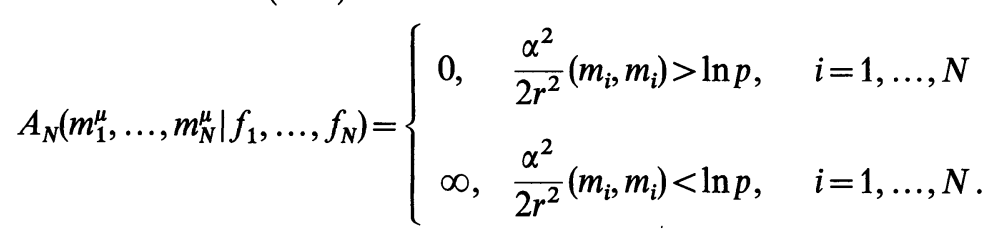

If

$$
\left(m_{i}, m_{i}\right)=2 \frac{r^{2}}{\alpha^{2}} \ln p, \quad i=1, \ldots, N,
$$

inserting (3.13) into the right-hand side of (3.12) we obtain the non-trivial limit

$$
\begin{aligned}
A_{N}\left(m_{1}^{\mu}, \ldots, m_{N}^{\mu} \mid f_{1}, \ldots, f_{N}\right)= & \int_{P^{1}\left(\mathbb{Q}_{p}\right)^{\times N}} f_{1}\left(x_{1}\right) d \mu_{0}\left(x_{1}\right) \ldots f_{N}\left(x_{N}\right) d \mu_{0}\left(x_{N}\right) \\
& \times \prod_{i<j}^{N} \mid x_{i}, x_{j}{\frac{\alpha}{r_{p}^{2}}}^{r^{2} p}\left(m_{i}, m_{j}\right)
\end{aligned}
$$


Here the scalar product $\left(m_{i}, m_{i}\right)=\sum_{\mu=1}^{D}\left(m_{i}^{\mu}\right)^{2}$.

Let us consider the generalized Bruhat-Tits tree $F_{g}$ with $g>0$. Using the relations (2.13)-(2.16), the decomposition (3.1) and the formulas (3.2), (3.3), (3.8), and (3.9) we obtain the relation (3.14). If the integers $m_{i}^{\mu}$ satisfy the conditions (2.10), (3.15) we have the non-trivial limit (3.10):

$$
\begin{aligned}
& A_{N}\left(m_{1}^{\mu}, \ldots, m_{N}^{\mu} \mid f_{1}, \ldots, f_{N}\right)=\sum_{\substack{\left\{x_{i}^{R}\right) \in F_{F_{0}^{R}}^{R} \\
b\left(x_{i}^{R}\right)>0}} W\left(\sum_{j=1}^{N} m_{j}^{\mu} x_{j}^{R}\right) \\
& \quad \times \int_{\mathbf{Z}_{p}\left[a_{0}<b\left(x_{1}^{R}\right)\right]} f_{1}\left(x_{1}^{R} \rightarrow x_{1}\right) d x_{1} \ldots \int_{\mathbf{z}_{p}\left[a_{0}<b\left(x_{N}^{R}\right)\right]} f_{N}\left(x_{N}^{R} \rightarrow x_{N}\right) d x_{N} \\
& \quad \times \prod_{i<j}^{N}\left|x_{i}-x_{j}\right|_{p} \frac{\alpha^{2}}{r^{2} \ln p}\left(m_{i}, m_{j}\right) \delta_{x_{i}^{R}, x_{j}^{R}}
\end{aligned}
$$

where the correlation function $W\left(\sum_{j=1}^{N} m_{j}^{\mu} x_{j}^{R}\right)$ is given by the equations (2.13)-(2.16). The proof of the formula (3.17) is exactly analogous to the case $g=0$.

Now we consider the limit $r \rightarrow \infty, m_{i}^{\mu} \rightarrow \infty$ such that

$$
\frac{m_{i}^{\mu}}{r} \rightarrow k_{i}^{\mu} \alpha^{-1}(\ln p)^{1 / 2}, \quad i=1, \ldots, N,
$$

where $k_{i}^{\mu}$ are real numbers satisfying, due to (2.10) and (3.15), the conditions

$$
\begin{array}{ll}
\sum_{i=1}^{N} k_{i}^{\mu}=0, & \mu=1, \ldots, D \\
\left(k_{i}, k_{i}\right)=2, & i=1, \ldots, N .
\end{array}
$$

The amplitudes (3.16) and (3.17) tend respectively to

$$
\begin{aligned}
& A_{N}\left(m_{1}^{\mu}, \ldots, m_{N}^{\mu} \mid f_{1}, \ldots, f_{N}\right)=\int_{P^{1}\left(\mathbb{Q}_{p}\right)^{\times N}} f_{1}\left(x_{1}\right) d \mu_{0}\left(x_{1}\right) \ldots f_{N}\left(x_{N}\right) d \mu_{0}\left(x_{N}\right) \\
& \times \prod_{i<j}^{N}\left|x_{i}, x_{j}\right|_{p}^{\left(k_{i}, k_{j}\right)}
\end{aligned}
$$

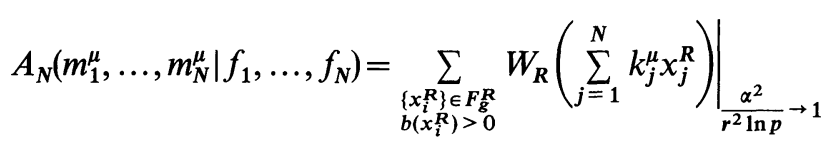

$$
\begin{aligned}
& \times \int_{\mathbb{Z}_{p}\left[a_{0}<b\left(x_{1}^{R}\right)\right]} f_{1}\left(x_{1}^{R} \rightarrow x_{1}\right) d x_{1} \cdots \int_{\mathbb{Z}_{p}\left[a_{0}<b\left(x_{N}^{R}\right)\right]} f_{N}\left(x_{N}^{R} \rightarrow x_{N}\right) d x_{N} \prod_{i<j}^{N}\left|x_{i}-x_{j}\right|_{p}^{\left(k_{i}, k_{j}\right) \delta_{x_{i}^{R}, x_{j}^{R}}},
\end{aligned}
$$

because of the $\theta$-function in (2.13) tends to 1 . Here the correlation function $\left.W_{R}\left(\sum_{j=1}^{N} k_{j}^{\mu} x_{j}^{R}\right)\right|_{\frac{\alpha^{2}}{r^{2} \ln p} \rightarrow 1}$ is given by the formula (2.14) with the change $\frac{\alpha^{2}}{r^{2}} \rightarrow \ln p$.

If $f_{j}(x) \rightarrow 1, j=1, \ldots, N$ the amplitude (3.19) coincides with the result of the paper [9]. If $f_{j}(x) \rightarrow 1, j=1, \ldots, N-3, f_{N-2}(x) d \mu_{0}(x) \rightarrow \delta(x-\infty) d x, \quad f_{N-1}(x) d \mu_{0}(x)$ $\rightarrow \delta(x-1) d x, f_{N}(x) d \mu_{0}(x) \rightarrow \delta(x) d x$, then the amplitude (3.19) coincides with the 
Freund-Olson amplitude [3]. The result (3.20) is new since the boundary $\partial F_{g}$ for the generalized Bruhat-Tits tree $F_{g}$ with $g>0$ was not computed explicitly in the paper [10].

Acknowledgements. The authors are grateful to A. Mironov and A. Zabrodin for useful discussions.

\section{References}

1. Volovich, I.V.: p-adic string. Class. Quant. Gravity 4, L83-L87 (1987)

2. Grossman, B.: p-Adic strings, the Weyl conjectures and anomalies. Phys. Lett. 197 B, 101-104 (1987)

3. Freund, P.G.O., Olson, M.: Non-archimedean strings. Phys. Lett. 199 B, 186-190 (1987)

4. Freund, P.G.O., Witten, E.: Adelic string amplitudes. Phys. Lett. 199 B, 191-194 (1987)

5. Gervais, J.-L.: p-Adic analyticity and Virasoro algebras for conformal theories in more than two dimensions. Phys. Lett. 201 B, 306-310 (1988)

6. Brekke, L., Freund, P.G.O., Olson, M., Witten, E.: Non-archimedean string dynamics. Nucl. Phys. B 302, 365-402 (1988)

7. Frampton, P.H., Okada, Y.: p-adic string $N$-point function. Phys. Rev. Lett. 60, 484-486 (1988)

8. Knizhnik, V.G., Polyakov, A.M.: Unpublished (1987)

Parisi, G.: On p-adic functional integral. Mod. Phys. Lett. A 3, 639-643 (1988)

Spokoiny, B.L.: Quantum geometry of non-archimedean particles and strings. Phys. Lett. 208 B, 401-406 (1988)

Zhang, R.B.: Lagrangian formulation of open and closed p-adic strings. Phys. Lett. 209 B, 229-232 (1988)

9. Zabrodin, A.: Non-archimedean strings and Bruhat-Tits trees. Commun. Math. Phys. 123, 463-483 (1989)

10. Chekhov, L., Mironov, A., Zabrodin, A.: Multiloop calculations in p-adic string theory and Bruhat-Tits trees. Commun. Math. Phys. 125, 675-711 (1989)

11. Mumford, D.: An analytic construction of degenerating curves over complete local rings. Compos. Math. 24, 129-174 (1972)

12. Manin, Yu.I.: p-adic automorphic functions. Sovr. Probl. Mat., Vol. 3, 5-92. Moscow: VINITI 1974 (in Russian)

Gerritzen, L., van der Put, M.: Schottky groups and Mumford curves. Berlin, Heidelberg, New York: Springer 1980

13. Bruhat, F., Tits, J.: Group reductifs sur un corps local. I. Données radicielles valuées. Publ. Math. IHES 41, 5-251 (1972)

14. Serre, J.P.: Trees. Berlin, Heidelberg, New York: Springer 1980

15. Zinoviev, Yu.M.: Lattice R-gauge theories. Theor. Math. Phys. 49, No. 2 (1981)

16. Friedan, D., Martinec, E., Shenker, S.H.: Nucl. Phys. B 271, 93 (1986)

17. Zinoviev, Yu.M.: Duality in the Abelian gauge lattice theories. Theor. Math. Phys. 43, N3, 481-490 (1980)

18. Wilson, K.G.: Confinement of quarks. Phys. Rev. D 10, 2445-2459 (1974)

19. Villain, J.: Theory of one- and two-dimensional magnets with an easy magnetization plane. II. The planar, classical, two-dimensional magnet. J. Phys. 36, 581-590 (1975)

20. Mumford, D.: Tata lectures on Theta I, II. Boston, Basel, Stuttgart: Birkhäuser 1983, 1984

Communicated by Ya. G. Sinai

Received November 11, 1989 